\title{
The Association of Pneumonia with Clinical Outcome in Patients with Inhalation Injury*
}

\author{
Sharmila Dissanaike ${ }^{1 \#}$, Stephen $\operatorname{Cox}^{2}$, Soleil Arrieta ${ }^{1}$ \\ ${ }^{1}$ Department of Surgery, Texas Tech University Health Sciences Center, Lubbock, USA \\ ${ }^{2}$ Research and Testing Laboratory, Lubbock, USA \\ Email: "sharmila.dissanaike@ttuhsc.edu
}

Received October 30, 2012; revised November 30, 2012; accepted December 9, 2012

\begin{abstract}
Introduction: Inhalation injury is a particularly lethal form of thermal burn injury, and is associated with increased morbidity and mortality. Pneumonia is a common complication of inhalation injury, due to the increased susceptibility of lungs that have been stripped of their biologic defense mechanisms, as well as the general susceptibility of the burn population to infections. While older series suggest that pneumonia is associated with worse mortality and morbidity, recent reports suggest that this may not be the case in all populations. Methods: We attempted to clarify the impact of pneumonia in terms of mortality, length of mechanical ventilation, need for tracheostomy, and discharge disposition, in patients admitted with inhalation injury by performing a retrospective review of patients admitted to a regional burn center 2002-2009. Burn registry and electronic chart review were used to obtain demographic, clinical and outcome data. Univariate and multivariate analysis was used to compare outcomes in patients who developed pneumonia versus those who did not. Results: The study cohort comprised 166 patients, of whom 21 (13\%) were diagnosed with pneumonia. Development of pneumonia was not predicted by age, surface area burned or other complications such as acute respiratory distress syndrome. Surprisingly, pneumonia was associated with reduced inpatient mortality $(p=0.006)$. However, patients who developed pneumonia were also more likely to have prolonged ventilator dependence (19 vs 5 days, $p<0.001)$, require intensive respiratory therapy $(p<0.001)$, receive tracheostomy $(p<0.001)$ and have an increased overall length of stay (33 vs. 10 days, $p<0.001$ ). They were significantly less likely to be discharged home and more likely to be transferred to a nursing facility or rehabilitation center upon discharge $(p=0.002)$.
\end{abstract}

Keywords: Inhalation Injury; Pneumonia; Burns; Ventilator Associated Pneumonia

\section{Introduction}

Up to a third of patients with major burns may have associated smoke inhalation injury, depending on the diagnostic criteria used. Inhalation injury continues to be associated with poor prognosis and significant mortality in the burn population. Improvements in critical care, fluid resuscitation and early excision and grafting of the burn wound that have led to substantial declines in mortality and morbidity from cutaneous burns have unfortunately not been replicated in the treatment of smoke inhalation [1].

Pneumonia is a very common complication of inhalation injury, with incidence rates varying from $27 \%-87 \%$, partly due to differing criteria and definitions of both pneumonia and inhalation injury in different centers [2,3]. Studies from previous decades tend to have higher incidence rates than more current reports [4,5]. There are several possible reasons for the high prevalence of pneu-

\footnotetext{
*The authors declare they have no competing interest.

${ }^{\#}$ Corresponding author.
}

monia among this population. Firstly, burn patients have long been recognized to be at significant risk for infection due to breaches of the skin protective barrier, as well as transient immunocompromise from the metabolic alterations of the burned state [6]. Similar to the destruction of the cutaneous barrier leading to an increase in invasive infections, the destruction of natural pulmonary defense mechanisms would be expected to result in an increase in the incidence of pneumonia. The pathophysiology of smoke inhalation injury involves the activation of an inflammatory cascade leading to free radical generation, which combines with toxic products of combustion to de-epithelialize the endobronchial tree, increase water permeability and inactivate surfactant, leading to the accumulation of fibrinous debris and plugs within the bronchial lumen. These plugs cause pulmonary atelectasis distal to their formation, worsen the ventilation/perfusion mismatch, and serve as platforms for bacterial colonization [7]. By simultaneously stripping the lungs of their protective mechanisms while creating wide-spread pockets of atelactasis, smoke inhalation creates ideal con- 
ditions for pneumonia to occur.

While the incidence of pneumonia is unquestionably higher in patients with smoke inhalation, the effects upon the patient's overall prognosis are less certain. Inhalation injury itself increases mortality and morbidity, but an incremental increase in mortality by the development of subsequent pneumonia has not been clearly demonstrated. Edelman et al. reported a doubling of mortality in their series of 117 patients with smoke inhalation; however this did not reach statistical significance [3]. Shirani et al. in a larger study of over 1000 patients demonstrated an increase in mortality with pneumonia of up to $40 \%$; however the standard of critical care of burns, respiratory failure and infectious disease have all undergone vast improvements since this cohort of patients was reported nearly 25 years ago [5].

In a general critical care population, ventilator associated pneumonia (VAP), a subset of pneumonia that occurs in patients who are ventilated over 48 hours, has been shown to double mortality and significantly increase both morbidity and cost of care [8]. A recent analysis described a 6\% attributable mortality to VAP [9]. However, in several studies limited to trauma patients, VAP has not been shown to increase mortality $[10,11]$. A recent series of 2436 trauma patients in 27 intensive care units from 9 countries showed an actual reduction in mortality in patients with VAP, suggesting that multiple factors are in effect in these situations, and that results obtained from a primarily medical population cannot be extrapolated to a trauma population [12].

Patients suffering burn and inhalation injury have many of the same attributes of patients suffering other traumatic injury - a high proportion of healthy young people suffering a sudden catastrophic event. However, there are significant differences in the level of metabolic derangement, with thermal and inhalation injury resulting in much greater fluid shifts and hypermetabolism than all but the most severe traumatic injuries. Therefore the results from the general trauma population cannot be directly extrapolated to burn victims. Since there are significantly fewer burn victims, and even fewer victims of inhalation injury, both nationwide and globally, compared to victims of mechanical trauma, there is less data on the effects of pneumonia in these patients compared to the general trauma population. This study investigates the consequences of pneumonia in burned patients with inhalation injury, specifically regarding mortality.

\section{Materials and Methods}

The Texas Tech University Health Sciences Center Institutional Review Board approved this study. Informed consent was not required due to the retrospective nature and use of de-identified data.

\subsection{Subject Selection}

A retrospective review was performed of all patients diagnosed with smoke inhalation injury admitted to the Timothy J. Harnar regional burn center, Lubbock, TX between January 2002 and December 2009. Subjects were identified using the burn registry and discharge coding information. Demographic and injury information, clinical parameters on admission, ventilator modes, respiratory therapy measures used and outcomes were abstracted from the burn registry and electronic and paper medical records.

\subsection{Definitions and Clinical Practice}

All smoke inhalation patients were admitted to the Timothy J. Harnar regional burn center. Care was under the direction of one of three attending burn surgeons and surgical residents working under their direction. Aggressive fluid resuscitation was performed according to the Parkland formula, modified for the addition of dextrose in children, and adjusted for urine output per standard protocols. Early enteral feeding was commenced in all patients. During the period of this study, sedation, pain control, and ventilator management of intubated patients was per attending surgeon discretion, as was the use of adjunctive respiratory treatments. The decision to perform tracheostomy was also per individual attending surgeon, but a policy to not perform early (within 2 weeks of admission) elective tracheostomy except in cases of concurrent severe traumatic brain injury was in place.

Smoke inhalation was defined by a combination of relevant clinical history including closed space fire, and physical signs and symptoms such as carbonaceous sputum, dyspnea, hoarseness or stridor. Positive bronchoscopic findings included erythema, edema, ulceration and carbonaceous particles. Current burn center protocol requires that all suspected smoke inhalation patients requiring mechanical ventilation undergo bronchoscopy shortly after admission; however during the early years of the study this protocol was not in place and bronchoscopy was based on attending physician discretion.

Pneumonia was defined using the criteria set by the National Trauma Data Bank: presence of fever, leukocytosis, Gram stain of sputum with a predominant organism and moderate to many white blood cells, chest radiograph with a pneumonic infiltrate, and culture of sputum demonstrating a pathogen. Fever was defined as $<96.8 \mathrm{~F}$ or $>102.2 \mathrm{~F}$, and leukocytosis as a WBC $>12$. All clinically suspected cases of pneumonia were confirmed by microbiologic data.

\subsection{Statistical Analysis}

Subjects were divided based on whether they had pneumonia or not, and analyzed with regard to outcome meas- 
ures. The primary outcome measure was in-hospital mortality; secondary outcome measures were length of stay, ventilator days, and need for tracheostomy. Covariates included the ventilator mode on admission, and respiratory therapies used. A Cox proportional hazards model was used to relate survival to pneumonia, multisystem organ failure (MSOF), Acute Respiratory Distress Syndrome (ARDS), sepsis, age and total body surface area burned (TBSA), and the proportional hazard assumption for all variables was testing using the method of Grambsch and Therneau [13]. By including all variables in the model simultaneously the intent was to estimate the unique effect of each variable while holding all other variables constant. Since time to onset of pneumonia was not available in our database, the analysis was repeated excluding all patients who died within the first 5 days i.e. those who did not live long enough to develop the clinical signs of pneumonia. A $p<0.05$ was considered significant for hypothesis testing. All analyses were performed using SPSS 18.0 (Chicago, IL) and R (Development Core Team, 2011) with the survival library [14] (Therneau and Lumley, 2011).

\section{Results}

Data was available on 166 patients with inhalation injury who were admitted during this time frame, of whom 123 were men (74\%). Regarding clinical characteristics on presentation, the majority of patients $(87,50 \%)$ had facial burns, 41 (24\%) had singed nasal mucosa, 38 (22\%) had difficulty swallowing and 33 (19\%) had carbonaceous sputum. 131 (80\%) were intubated and mechanically ventilated on arrival or in the emergency room, and 110 (66\%) had bronchoscopy performed on admission.

Assist control was the initial mode of ventilation in the majority 65 (61\%) of patients, with $18(17 \%)$ placed on high frequency oscillatory ventilation, 14 (13\%) on synchronized intermittent mandatory ventilation, and 9 (8\%) on airway pressure release ventilation. Airway pressure release was used as rescue therapy for hypoxia in 27 patients (25\%), and high frequency oscillatory ventilation in $8(7 \%)$.

Adjunctive respiratory therapies were used in a minority of patients, predominantly after a diagnosis of pneumonia ( $p=0.001)$. Cough stimulation was used in 25 (15\%), chest physiotherapy in 41 (25\%), and kinetic /rotation therapy in 10 (6\%) smoke inhalation patients.

Pneumonia was diagnosed in 21 patients (13\%). The demographics and risk factors of patients with pneumonia compared to those who did not develop pneumonia are shown in Table 1. Since the groups were not normally distributed within themselves with regard to several variables, the median values and interquartile range are displayed, rather than the mean and standard deviation. While the mean TBSA did not differ between
Table 1. Baseline characteristics and initial ventilator mode used, by factor (pneumonia).

\begin{tabular}{cccc}
\hline & $\begin{array}{c}\text { Without } \\
\text { pneumonia }\end{array}$ & Pneumonia & Test statistic \\
\hline & $N=129$ & $N=21$ & \\
Age & $26,45,57$ & $25,32,53$ & $F_{1,148}=0.74, p=0.392^{1}$ \\
Sex & $74 \%(96)$ & $76 \%(16)$ & $\chi_{1}^{2}=0.03, p=0.863^{2}$ \\
Race & & & \\
Hispanic & $37 \%(46)$ & $42 \%(8)$ & $\chi_{3}^{2}=2.07, p=0.557^{2}$ \\
White & $58 \%(73)$ & $47 \%(9)$ & \\
Black & $4 \%(5)$ & $11 \%(2)$ & \\
Other & $1 \%(1)$ & $0 \%(0)$ & \\
Body mass index & $22.2,26.6$, & $22.5,25.2$, & $F_{1,124}=0.95, p=0.332^{1}$ \\
(BMI) & 31.8 & 27.4 & \\
Total burned & & & \\
surface area & $0,6.25,35$ & $4,21,32$ & $F_{1,141}=1.53, p=0.218^{1}$ \\
(TBSA) & & & \\
Intubated & $78 \%(100)$ & $100 \%(21)$ & $\chi_{1}^{2}=5.66, p=0.017^{2}$ \\
Initial PO ${ }_{2}:$ FiO \\
ratio & 3.50 & 3.38 & $F_{1,126}=0.33, p=0.567^{1}$ \\
Initial & & & \\
ventilator mode & & & $\chi_{6}^{2}=9.71, p=0.137^{2}$ \\
AC & $44 \%(53)$ & $44 \%(8)$ & \\
SIMV & $9 \%(11)$ & $22 \%(4)$ & \\
CPAP & $1 \%(1)$ & $0 \%(0)$ & \\
APRV & $6 \%(7)$ & $11 \%(2)$ & \\
HFOV & $12 \%(14)$ & $22 \%(4)$ & \\
\hline
\end{tabular}

Ventilator modes are: assist control (AC), synchornized intermittent mandatory ventilation (SIMV), continous positive aurway pressure (CPAP), airway pressure release ventilation (APRV) and high frequency oscillatory ventilation (HFOV). a, b, c represent the lower quartile a, the median b, and the upper quartile $c$ for continuous variables. Numbers after percents are frequencies. Tests used: ${ }^{1}$ Wilcoxon test; ${ }^{2}$ Pearson test.

groups, (22.5 vs. 21.3, $p=0.859)$, there was an uneven distribution with more patients in the "no pneumonia" group admitted with pure inhalation injury and no cutaneous burns. Development of pneumonia was not related to age, total body surface area (TBSA) burned, initial ventilator mode used or the development of acute respiratory distress syndrome (ARDS). Outcome measures for patients with pneumonia are shown in Table 2. Patients who developed pneumonia were significantly more likely to receive tracheostomy ( $p<0.001$ ), have prolonged ventilator dependence (19 vs. 5 days, $p<0.001$ ) and an increased overall length of stay (33 vs. 10 days, $p<0.001$ ). Patients who developed pneumonia were significantly less likely to be discharged home and more likely to be transferred to a nursing facility or rehabilitation center upon discharge from the burn center $(p=0.002)$.

In the global model, pneumonia was associated with a significant reduction in mortality $(p=0.006)$, albeit with a much reduced effect size (Figure 1). Age $(p=0.011)$ and TBSA $(p<0.001)$ significantly increased mortality, 
Table 2. Outcomes by factor(pneumonia).

\begin{tabular}{|c|c|c|c|}
\hline & Without pneumonia & Pneumonia & Test Statistic \\
\hline & $N=129$ & & \\
\hline & $\chi_{1}^{2}=5.1, p=0.024^{2}$ & $N=21$ & \\
\hline $\begin{array}{l}\text { Days on } \\
\text { Ventilator }\end{array}$ & $0,2,6$ & $12,13,20$ & $\begin{array}{l}F_{1,148}=36.92, \\
p<0.001^{1}\end{array}$ \\
\hline Length of Stay & $2,5,14$ & $15,25,53$ & $\begin{array}{l}F_{1,148}=37.24, \\
p<0.001^{1}\end{array}$ \\
\hline Tracheostomy & $9 \%(12)$ & $52 \%(11)$ & $\begin{array}{l}\chi_{1}^{2}=25.31 \\
p<0.001^{2}\end{array}$ \\
\hline $\begin{array}{l}\text { Rotation } \\
\text { Therapy }\end{array}$ & $7 \%(7)$ & $27 \%(3)$ & $\begin{array}{l}\chi_{1}^{2}=4.65 \\
p=0.031^{2}\end{array}$ \\
\hline $\begin{array}{c}\text { Chest } \\
\text { Physiotherapy }\end{array}$ & $25 \%(27)$ & $88 \%(14)$ & $\begin{array}{l}\chi_{1}^{2}=25.22 \\
p<0.001^{2}\end{array}$ \\
\hline $\begin{array}{l}\text { Cough } \\
\text { Stimulation }\end{array}$ & $14 \%(15)$ & $62 \%(10)$ & $\begin{array}{l}\chi_{1}^{2}=21.23 \\
p<0.001^{2}\end{array}$ \\
\hline ARDS & $16 \%(20)$ & $21 \%(4)$ & $\begin{array}{l}\chi_{1}^{2}=0.36 \\
p=0.55^{2}\end{array}$ \\
\hline MSOF & $20 \%(25)$ & $26 \%(5)$ & $\begin{array}{l}\chi_{1}^{2}=0.47 \\
p=0.494^{2}\end{array}$ \\
\hline \multicolumn{4}{|l|}{ Disposition } \\
\hline Home & $54 \%(70)$ & $57 \%(12)$ & $\begin{array}{l}\chi_{2}^{2}=12.91 \\
p=0.002^{2}\end{array}$ \\
\hline Nursing Facility & $6 \%(8)$ & $29 \%(6)$ & \\
\hline Death & $40 \%(51)$ & $14 \%(3)$ & $\begin{array}{l}\chi_{1}^{2}=5.1 \\
p=0.024^{2}\end{array}$ \\
\hline
\end{tabular}

a, b, c represent the lower quartile a, the median b, and the upper quartile c for continuous variables. Numbers after percents are frequencies. Tests used: ${ }^{1}$ Wilcoxon test; ${ }^{2}$ Pearson test.

with TBSA having the strongest impact. The effects of MSOF and ARDS were not significant once other covariates were considered. When patients who died within the first 5 days were excluded, the association remained significant, suggesting that this effect was not due to lead-time bias alone (data not shown). The KaplanMeier survival curve is shown in Figure 2, demonstrating the improved survival for patients with pneumonia compared to those who did not develop pneumonia.

The organisms that were present in patients with pneumonia are shown in Figure 3. There was a trend toward increased mortality with Pseudomonas aeruginosa infection but small sample size prevented meaningful statistical analysis of the association between organism and outcome.

\section{Discussion}

This study demonstrates that pneumonia did not worsen inpatient mortality in a cohort of patients with inhalation injury. In fact, pneumonia was associated with improved survival in this high-risk group, a surprising finding that nevertheless does have some parallels with reports on trauma patients with pneumonia. While it is very unlikely that this is a causal relationship, it is possible that the development of pneumonia serves as a marker for patients with improved survival, and raises further questions to be explored.

This phenomenon could partly be explained by survivor-time bias; patients who died early in their hospital admission simply did not have time to develop pneumonia prior to expiring from other causes. Since the existing literature suggests that a large proportion of patients with inhalation injury will develop pneumonia, it is plausible to assume that early deaths would skew the data toward the results shown. Unfortunately we were unable to determine the exact date of initial diagnosis of pneumonia using the current dataset; therefore it is possible that survivor-time bias partially accounts for the findings. However since the association of pneumonia with improved survival remained consistent even after excluding patients who died within the first 5 days, the authors believe that this does not account for the strong association demonstrated.

A biologic explanation is also plausible; the clinical definition of pneumonia requires a relatively vigorous host immune response, which may indicate underlying genetic or biologic traits that portend an improved prognosis. Davis et al. [15] demonstrated that patients who succumbed to their inhalation injury had lower bronchoalveolar lavage fluid concenterations of numerous immunomodulators, including C5a, interleukin 1beta, IL1 RA, IL-2, IL-8, IL-10 and IL-13, and TNF-alpha. This study suggests that an early immune hyporesponsiveness to smoke inhalation is associated with increased mortality. Conversely, patients who mount an aggressive host response - and are more likely to manifest signs and symptoms consistent with pneumonia-may have an improved survival, as was demonstrated in this study. Larger multi-center studies of patients with smoke inhalation would be very valuable in elucidating the relationship between pneumonia, other morbidity, and survival.

Although pneumonia was associated with improved survival, it also significantly increased resource utilization, consistent with what has been demonstrated previously [16-18]. Patients who developed pneumonia had significantly longer length of time on ventilators, higher utilization of respiratory care services, longer hospital length of stay, and were more likely to require tracheostomy, all of which translate into a significant increase in cost of care. Therefore efforts to prevent pneumonia in this vulnerable population should continue, as there is a significant increase in morbidity and health care costs, if not mortality from this complication.

One issue of debate and concern in this area of research has been the potential for over-diagnosis of pneumonia in burned patients. Pham et al. [19] have shown that clinical criteria such as the clinical pulmonary 


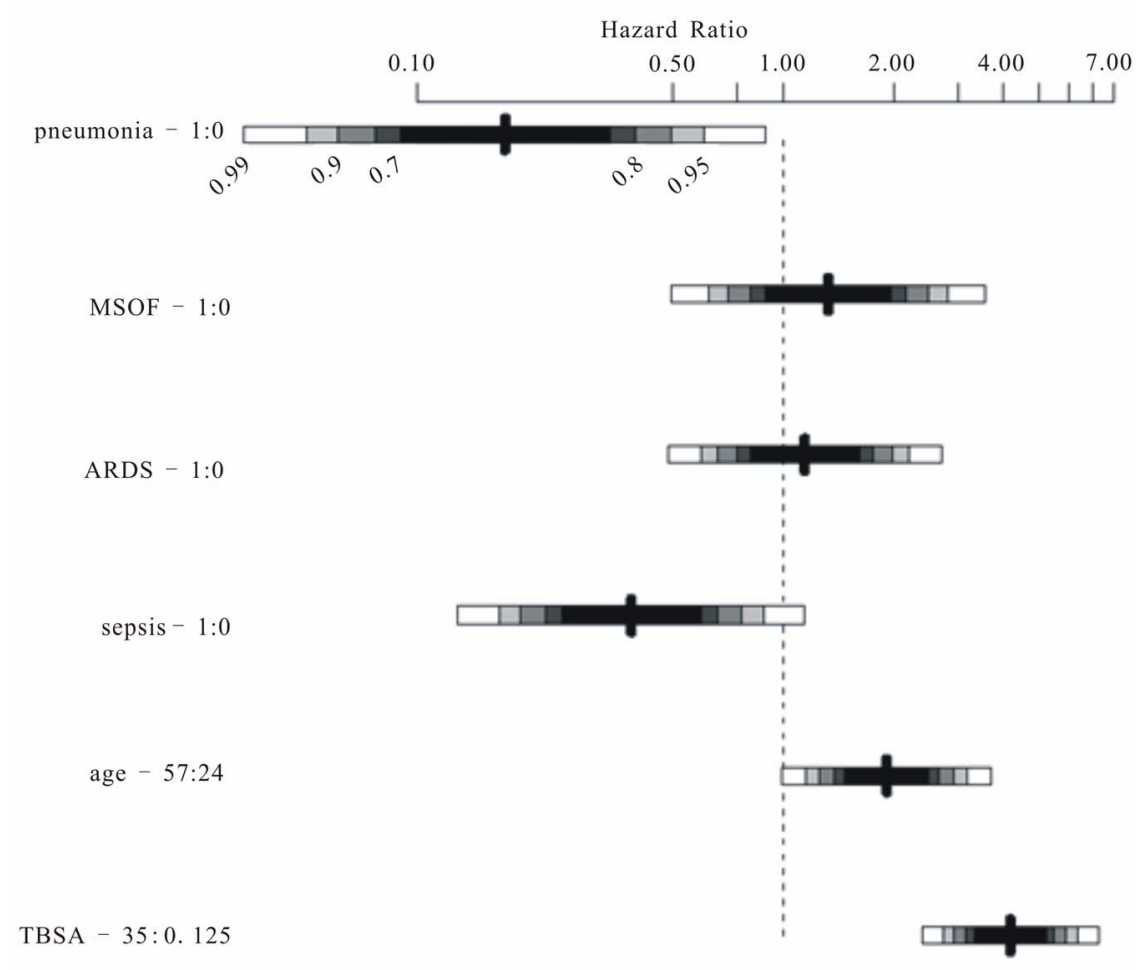

Figure 1. Effect sizes (hazard ratios) and confidence intervals illustrating the impacts of pneumonia, MSOF, ARDS, sepsis, age and TBSA on patient mortality. $95 \%$ confidence intervals are denoted by the light gray bounds around hazard ratio estimates, and all estimates are derived from the Cox proportional hazards model including all variables.

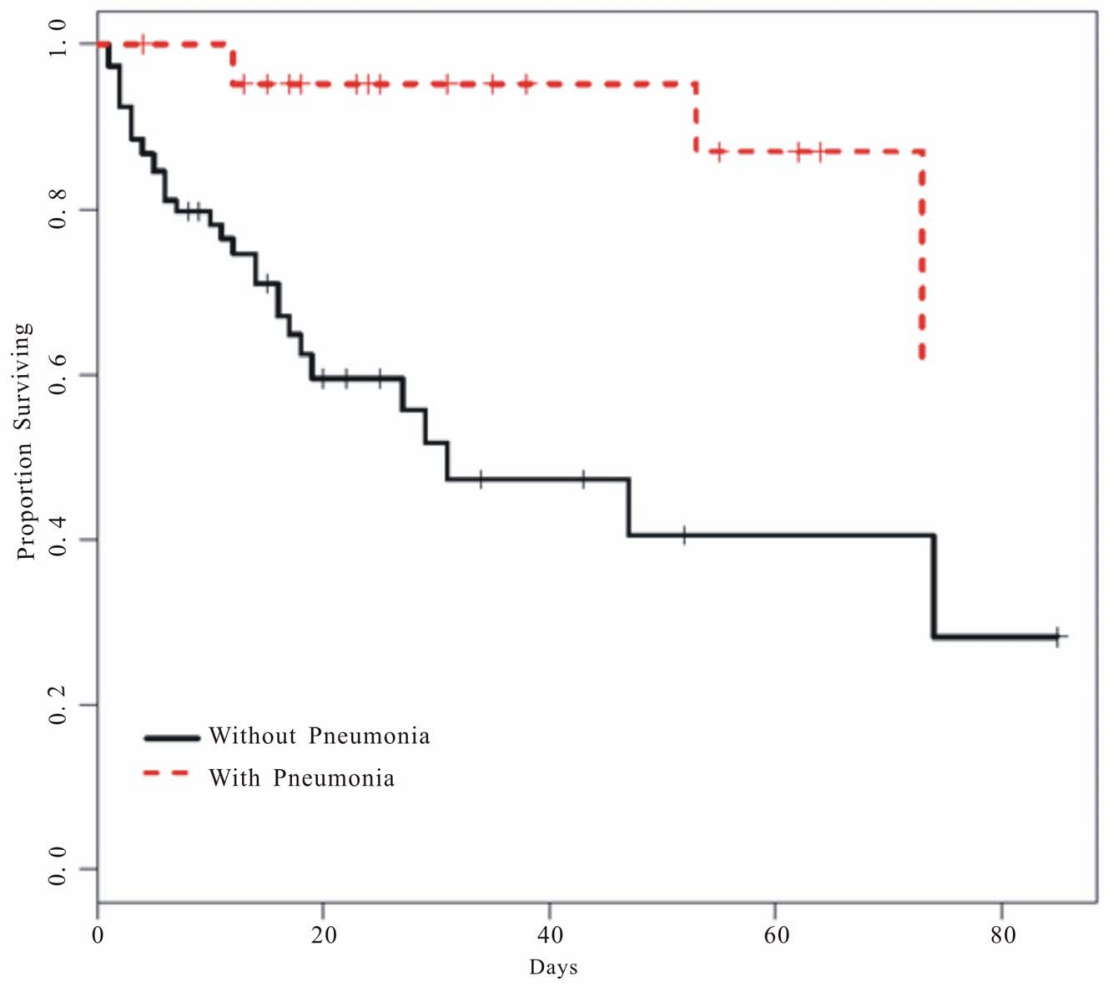

Figure 2. Patient survival as estimated by the Cox proportional hazards model. Here, survival is represented for patients with, and without, pneumonia, and is adjusted for all other covariates. Censored observations are denoted by the plus $(+)$ symbols on the survival curves. 


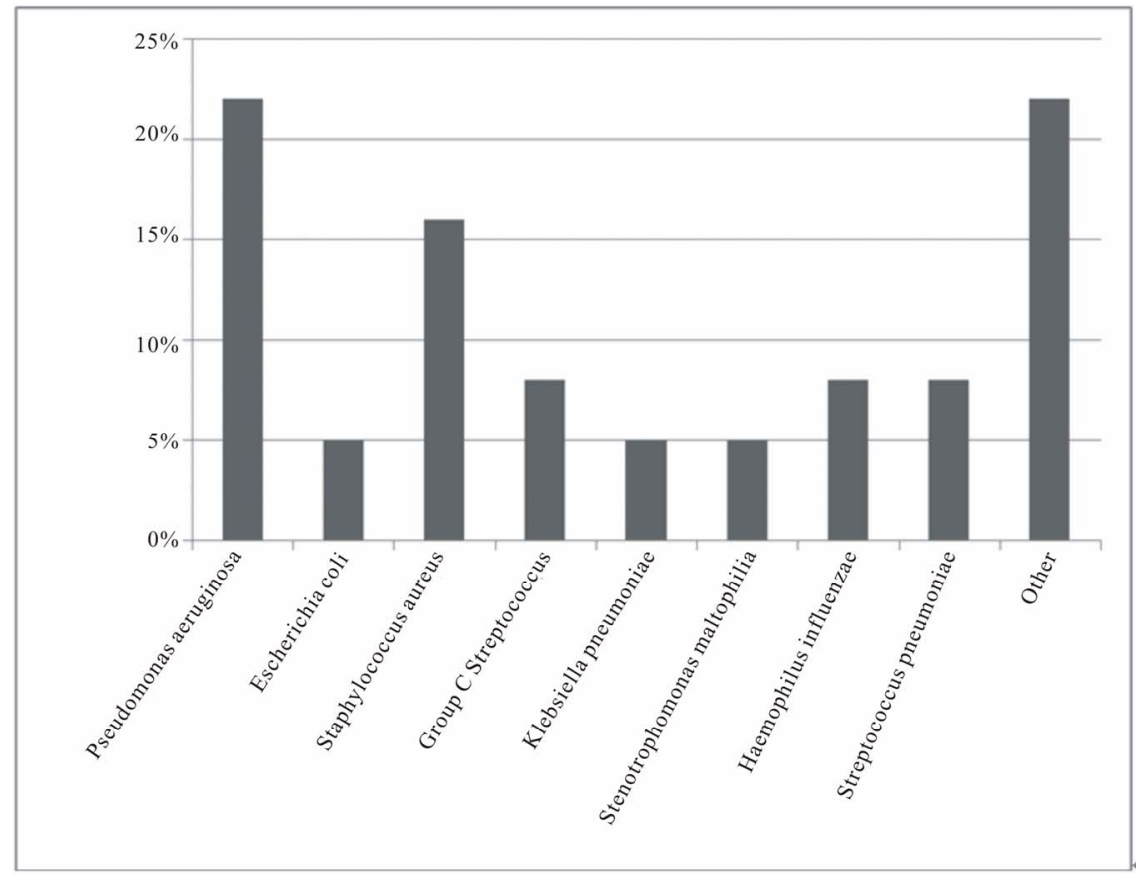

Figure 3. Bacterial species associated with pneumonia, shown as a percentage of total microbiologic types recorded.

infection score (CPIS) alone have limited utility in burned patients, and that clinical suspicion should be followed with quantitative microbiologic data. Diagnoses of pneumonia based on clinical markers alone are likely to result in a significant number of false positives, given the high prevalence of classic inflammatory markers such as pyrexia and leukocytosis among thermally injured patients, and pulmonary alterations such as copious bronchial secretions which may result from smoke inhalation alone, even without concurrent infection. Therefore current recommendations include obtaining microbiologic data in addition to clinical signs and symptoms for the diagnosis of pneumonia in burned patients [20]. All clinically suspected cases of pneumonia at our institution receive microbiologic analysis of sputum specimens; since 2003 this has been performed using BAL techniques and quantitative or semi-quantitative cultures. This policy is likely the reason for the relatively low rate (13\%) of pneumonia reported in this study, compared to older reports in patients with inhalation injury. The authors are confident that this cohort represents a "true positive", rather than an overdiagnosis from either the hypermetabolic burn response or contamination of sputum samples.

In addition to the drawbacks inherent in a retrospective review, there are several other limitations of this study. One drawback relates to the difficulty of classifying inhalation injury, pneumonia, and sepsis in the burned population. This study did not attempt to classify the severity of inhalation injury; a grading scheme based on bronchoscopic scoring has been described [21], but no uniformly accepted standard currently exists. The definition of pneumonia in burned patients has undergone some changes since the publication of the American Burn Association consensus statement in 2007 [20], which recommends 2 of the following be present for the diagnosis of pneumonia: chest radiograph changes, sepsis or change in sputum in concert with microbiologic data. The definition of sepsis in burned patients is also different to that of the regular population. Since the study period commenced several years before these definitions became available, the definitions used do not coincide with current practice. However the definitions used were uniform for the entire study period and population. As with any study that spans several years in an intensive care setting, there have been changes in practice. All patients suspected of inhalation injury now undergo bronchoscopy immediately upon admission, for both diagnostic and therapeutic indications. All patients suspected of pneumonia have bronchoalveolar lavage performed with protected specimens obtained for quantitative microbiologic analysis; these protocols had not been implemented at the outset of the study. While these variations in practice do reduce the internal consistency of the population, the authors do not believe that it negates the findings of the study. Comorbidities such as chronic obstructive pulmonary disease, history of tobacco use, coronary artery disease and congestive heart failure were not included in the analysis; certainly these and other medical conditions might have affected patient outcomes, both for the development of pneumonia as well as mortality. Finally, we did not separately analyze patients who fit the definition 
of ventilator associated pneumonia, due to the small number of total subjects, preferring to classify all positive cases as a form of healthcare-associated pneumonia complicating smoke inhalation.

\section{Conclusion}

Pneumonia may not increase mortality in patients with inhalation injury, although it does lead to a large increase in resource utilization and a prolonged hospitalization. The association of pneumonia with other clinical outcomes in inhalation injury patients deserves further detailed exploration.

\section{Acknowledgements}

The authors wish to thank the staff of the Timothy J. Harnar Burn Center for their excellent care of our patients, Benjamin Schaeffer MD for his assistance with data collection, the University Medical Center Trauma and Burn Services Office for their assistance with Burn Registry data, and the Texas Tech University Health Sciences Center Clinical Research Institute for their invaluable assistance with this study.

\section{REFERENCES}

[1] S. F. Miller, P. Bessey, C. W. Lentz, J. C. Jeng, M. Schurr and S. Browning, "National Burn Repository 2007 Report: A Synopsis of the 2007 Call for Data,” Journal of Burn Care \& Research, Vol. 29, No. 6, 2008, pp. 862-870. doi:10.1097/BCR.0b013e31818cb046

[2] N. Brusellaers, et al., "Burns, Inhalation Injury and Ventilator Associated Pneumonia: Value of Routine Surveillance cultures,” Burns, Vol. 38, No. 3, 2011, pp. 364-370.

[3] D. A. Edelman, N. Khan, K. Kempf and M. White, "Pneumonia after Inhalation Injury," Journal of Burn Care \& Research, Vol. 28, No. 2, 2007, pp. 241-246.

[4] L. W. Rue III, W. G. Cioffi, A. D. Mason, W. F. McManus and B. A. Pruitt Jr., "Improved Survival of Burned Patients with Inhalation Injury,” Archives of Surgery, Vol. 128, No. 7, 1993, pp. 772-778. doi:10.1001/archsurg.1993.01420190066009

[5] K. Z. Shirani, B. A. Preuitt, A. D. Mason Jr., "The Influence of Inhalation Injury and Pneumonia on Burn Mortality,” Annals of Surgery, Vol. 205, No. 1, 1987, pp. 82-87. doi:10.1097/00000658-198701000-00015

[6] R. Wurtz, et al., "Nosocomial Infections in a Burn Intensive Care Unit,” Burns, Vol. 21, No. 3, 1995, pp. 181-184. doi:10.1016/0305-4179(95)80005-9

[7] D. L. Traber, M. O. Maybauer and D. M. Maybauer, et al., "Inhalational and Acute Lung Injury," Shock, Vol. 24, Suppl. 1, 2005, pp. 82-87. doi:10.1097/01.shk.0000191338.39154.73

[8] N. Safdar, C. Dezfulian, F. Colardyn and S. Blot, "Clinical and Economic Consequences of Ventilator Associated Pneumonia: A Systematic Review," Critical Care Medicine, Vol. 33, No. 10, 2005, pp. 2184-2193.

\section{doi:10.1097/01.CCM.0000181731.53912.D9}

[9] J. F. Timsit, J. R. Zahar and S. Chevret, "Attributable Mortality of Ventilator-Associated Pneumonia," Current Opinion in Critical Care, Vol. 17, No. 5, 2011, pp. 464471. doi:10.1097/MCC.0b013e32834a5ae9

[10] M. E. Stone Jr., D. Snetman, A. O’Neill, J. Cucuzzo, J. Lindner, S. Ahmad and S. Teperman, "Daily Multidisciplinary Rounds to Implement the Ventilator Bundle Decreases Ventilator-Associated Pneumonia in Trauma Patients: But Does It Affect Outcome?” Surgical Infections, Vol. 12, No. 5, 2011, pp. 373-378. doi:10.1089/sur.2010.067

[11] A. Cook, S. Norwood and J. Berne, "Ventilator Associated Pneumonia Is More Common and of Less Consequence in Trauma Patients Compared with Other Critically Ill Patients,” Journal of Trauma, Vol. 69, No. 5, 2010, pp. 1083-1091. doi:10.1097/TA.0b013e3181f9fb51

[12] M. Margret, et al., "Ventilator Associated Pneumonia in Trauma Patients Is Associated with Lower Mortality: Results from EU-VAP Study,” Journal of Trauma, Vol. 69, No. 4, 2010, pp. 849-854. doi:10.1097/TA.0b013e3181e4d7be

[13] P. Grambsch and T. Therneau, "Proportional Hazards Tests and Diagnostics Based on Weighted Residuals," Biometrika, Vol. 81, No. 3, 1994, pp. 515-526. doi:10.1093/biomet/81.3.515

[14] T. Therneau and T. Lumley, "Survival: Survival Analysis, Including Penalized Likelihood,” 2011. http://CRAN.R-project.org/package=survival

[15] C. S. Davis, J. M. Albright, S. R. Carter, L. Ramirez, H. Kim, R. L. Gamelli and E. J. Kovacs, "Early Pulmonary Immune Hyporesponsiveness Is Associated with Mortality after Burn and Smoke Inhalation Injury," Journal of Burn Care \& Research, Vol. 33, No. 1, 2012, pp. 26-35. doi:10.1097/BCR.0b013e318234d903

[16] S. Hugonnet, P. Eggimann, F. Borst, P. Maricot, J. C. Chevrolet and D. Pittet, "Impact of Ventilator-Associated Pneumonia on Resource Utilization and Patient Outcome," Infection Control and Hospital Epidemiology, Vol. 25, No. 12, 2004, pp. 1090-1096. doi:10.1086/502349

[17] D. K. Warren, S. J. Shukla, M. A. Olsen, M. H. Kollef, C. S. Hollenbeak, M. J. Cox, M. M. Cohen and V. J. Fraser, "Outcome and Attributable Cost of Ventilator-Associated Pneumonia among Intensive Care Unit Patients in a Suburban Medical Center,” Critical Care Medicine, Vol. 31, No. 5, 2003, pp. 1312-1317. doi:10.1097/01.CCM.0000063087.93157.06

[18] J. G. Muscedere, C. M. Martin and D. K. Heyland, “The Impact of Ventilator-Associated Pneumonia on the Canadian Health Care System,” Journal of Critical Care, Vol. 23, No. 1, 2008, pp. 5-10. doi:10.1016/j.jcrc.2007.11.012

[19] T. N. Pham, M. J. Neff, J. M. Simmons, N. S. Gibran, D. M. Heimbach and M. B. Klein, "The Clinical Pulmonary Infection Score Poorly Predicts Pneumonia in Patients with Burns,” Journal of Burn Care \& Research, Vol. 28, No. 1, 2007, pp. 76-79. doi:10.1097/BCR.0b013E31802C88DB

[20] D. G. Greenhalgh, J. R. Saffle, J. H. Holmes IV, R. L. Gamelli, T. L. Palmieri, J. W. Horton, R. G. Tompkins, D. 
L. Traber, D. W. Mozingo, E. A. Deitch, C. W. Goodwin, D. N. Herndon, J. J. Gallagher, A. P. Sanford, J. C. Jeng, D. H. Ahrenholz, A. N. Neely, M. S. O’Mara, S. E. Wolf, G. F. Purdue, W. L. Garner, C. J. Yowler and B. A. Latenser, "American Burn Association Consensus Conference to Define Sepsis and Infection in Burns,” Journal of Burn Care \& Research, Vol. 28, No. 6, 2007, pp. 776-

\section{Abbreviations}

VAP: ventilator associated pneumonia;

WBC: white blood cell count;

MSOF: multi system organ failure;

TBSA: total body surface area;
790. doi:10.1097/BCR.0b013e3181599bc9

[21] F. W. Endorf and R. L. Gamelli, "Inhalation Injury, Pulmonary Perturbations, and Fluid Resuscitation,” Journal of Burn Care \& Research, Vol. 28, No. 1, 2007, pp. 8083. doi:10.1097/BCR.0B013E31802C889F

ARDS: acute respiratory distress syndrome;

IL: interleukin;

TNF: tumor necrosis factor;

CPIS: clinical pulmonary infection score. 